\title{
A Generic Mean Field Convergence Result for Systems of Interacting Objects
}

\author{
Jean-Yves Le Boudec \\ EPFL, I\&C \\ Lausanne, Switzerland \\ jean-yves.leboudec@epfl.ch
}

\author{
David McDonald \\ University of Ottawa \\ Department of Mathematics \\ dmdsg@uottawa.ca
}

\author{
Jochen Mundinger \\ EPFL, I\&C \\ Lausanne, Switzerland \\ jochen.mundinger@epfl.ch
}

\begin{abstract}
We consider a model for interacting objects, where the evolution of each object is given by a finite state Markov chain, whose transition matrix depends on the present and the past of the distribution of states of all objects. This is a general model of wide applicability; we mention as examples: TCP connections, HTTP flows, robot swarms, reputation systems. We show that when the number of objects is large, the occupancy measure of the system converges to a deterministic dynamical system (the "mean field") with dimension the number of states of an individual object. We also prove a fast simulation result, which allows to simulate the evolution of a few particular objects imbedded in a large system. We illustrate how this can be used to model the determination of reputation in large populations, with various liar strategies.
\end{abstract}

\section{Introduction}

We consider models in computer or communication systems where a large number of objects interact, and we are interested in going from a detailed (microscopic) description of the system at the object level to a global (macroscopic) description.

To this end, we define a generic model for interacting objects. Time is discrete. The evolution of each object is given by a finite state Markov chain, whose transition matrix depends on the present and the past of the distribution of states of all objects. In some form or another, this framework has been applied to a variety of cases, ranging from TCP connections [17, 2], HTTP flows [3], bandwidth sharing between streaming and file transfers [10], to robot swarms [11] and transportation systems [1]. In Section 3 we define the model and demonstrate its applicability on a few selected examples ranging in complexity from simple, memoryless, models to multiclass models with memory.
Next, our main result is that, as the number of objects becomes large, the model can be approximated by a deterministic equation, called the "mean field" (Theorem 4.1). We prove this approximation as an almost sure convergence result. This both provides a theoretical justification for the common practice of deterministic approximation, and, perhaps more importantly, a systematic method to derive such an approximation. Further, we also prove a fast simulation result (Theorem 5.1), which allows the simulation of the evolution of a few particular objects imbedded in a large system. This allows the computation, theoretically or by simulation, of such quantities as the time until a specified change of set occurs for a particular object starting in any arbitrary initial conditions. In Section 6, we show how to apply our modelling framework to a large, complex example (a reputation system). We show how our modelling approach can be used to assess the impact of different liar strategies. This section also serves as an illustration of the different steps involved in going from a free text description of the system to the application of the mean field convergence theorems.

Our model and results belong to a large family of mean field convergence results. The phrase "mean field" is often found in the literature, sometimes with slightly different meanings. The mean-field idea was first introduced in physics and has been used in the context of Markov process models of systems like plasma and dense gases where interaction between particles is somewhat weak, meaning that the strength of the interaction is inverse proportional to the size of the system. A given particle is seen as under a collective force generated by the other particles, similar to our model, but in a continuous time and space setting, and the interaction is usually assumed to depend on the first or second moment of the distribution of particles. In contrast, in this paper, we consider an arbitrary dependence. In [18] the mean field refers to making an independence assumption for one side of the master equation of a Markov process. In recent years, the mean-field approach has also found an appeal in the area of communication networks. To name but a few of the many publications, the reader is referred to 
[9], [16], [8] and [7]. Perhaps closest to our model are [4], which uses a continuous time limit, and [5], which uses a combination of discrete and continuous time and assumes a perfectly exchangeable system (i.e. all objects are i.i.d., whereas in our fast simulation result, objects may have different initial conditions).

\section{Main Notation List}

$\begin{array}{ll}d & \text { dimension of } \vec{r} \\ \mathcal{E}=\{1, \ldots, S\} & \begin{array}{l}\text { set of states for one object } \\ \text { function used to update the memory }\end{array} \\ K_{i, j}(\vec{r}) & \text { limit of } K_{i, j}^{N}(\vec{r}) \text { for large } N \text { or, } \\ & \text { value of } K_{i, j}^{N}(\vec{r}) \text { when independent of } N \\ K_{i, j}^{N}(\vec{r}) & \text { transition probability from } i \text { to } j \\ \vec{M}^{N}(t) & \text { occupancy measure (random process) } \\ M_{i}^{N}(t) & \text { the } i \text { th component of } \vec{M}^{N}(t) \\ & \text { proportion of objects in state } i \\ \vec{m} \in \mathbb{P}(\mathcal{E}) & \text { a possible value of } \vec{M}^{N}(t) \\ \vec{\mu}(t) & \text { a deterministic approximation of } \vec{M}^{N}(t) \\ N \in \mathbb{N} & \text { number of objects } \\ \mathcal{P}() & \text { same as } \mathcal{P}^{N}() \text { when } K(\vec{r}) \text { is used } \\ & \text { instead of } K^{N}(\vec{r}) \\ \mathcal{P} & \text { program that computes the next state } \\ & \text { for an object in state } i \text { when the memory is } \\ \vec{r} \text { and } u \text { is random uniform in }[0,1] & \text { memory (random process) } \\ \vec{R}^{N}(t) & \text { a possible value of } \vec{R}^{N}(t) \\ \vec{r} \in \mathbb{R}^{d} & \text { a deterministic approximation of } \vec{R}^{N}(t) \\ \vec{\rho}(t) & \text { number of possible states for one object } \\ S \in \mathbb{N} & \text { time } \\ t \in \mathbb{N} & \text { state of object } i \text { at time } t \\ X_{n}^{N}(t) & \end{array}$

\section{A Model of Interacting Objects with Indi- vidual State and Global Memory}

\subsection{Definition}

We consider a system of $N$ interacting objects. An object has a state $i$ in the finite set $\mathcal{E}=\{1, \ldots, S\}$. Time is discrete. Let $X_{n}^{N}(t), n=1 \ldots N$, be the state of object $n$ at time $t$. Also define the "occupancy measure" $\vec{M}^{N}(t)$ as the row vector whose $i$ th coordinate $M_{i}^{N}(t)$ is the proportion of objects that are in state $i$ at time $t$ :

$$
M_{i}^{N}(t)=\frac{1}{N} \sum_{n=1}^{N} 1_{\left\{X_{n}^{N}(t)=i\right\}}
$$

where $1_{\{x=i\}}$ is equal to 1 when $x=i$ and 0 otherwise.
At every time step, objects interact with their environment and may undergo a state transition. This model assumes that an object's state transition depends only on this object's current state and some function $\vec{R}^{N}(t) \in \mathbb{R}^{d}$ (where $d$ is some fixed integer) of the history of the occupancy measure.

More precisely, call $\mathbb{P}(\mathcal{E})$ the set of possible occupancy measures, i.e. $\mathbb{P}(\mathcal{E})$ is the set of nonnegative row vectors $\vec{\mu} \in \mathbb{R}^{S}$ that sum to 1 . We assume that there is a continuous (deterministic) mapping $g(): \mathbb{R}^{d} \times \mathbb{P}(\mathcal{E}) \rightarrow \mathbb{R}^{d}$ such that the following update equation holds, for $t \geq 0$

$$
\vec{R}^{N}(t+1)=g\left(\vec{R}^{N}(t), \vec{M}^{N}(t+1)\right)
$$

The initial value $\vec{R}^{N}(0)$ is chosen according to some probability distribution on $\mathbb{R}^{d}$. With an appropriate choice of $d$ and of the mapping $g(), \vec{R}^{N}(t)$ represents the memory of the occupancy measure (see below for examples).

State transitions for individual objects are assumed to satisfy the following rules. The next state for an object depends only on its current state and the value of the global memory $\vec{R}^{N}(t+1)$ : for all objects $n$

$$
\begin{aligned}
& \mathbb{P}\left\{X_{n}^{N}(t+1)=j \mid \vec{R}^{N}(t)=\vec{r}, X_{n}^{N}(t)=i\right\} \\
& =K_{i, j}^{N}(\vec{r})
\end{aligned}
$$

It is possible for an object not to do any transition during one time step (this may occur when $K_{i, i}^{N}(\vec{r})>0$ ). The $S \times S$ matrix $K^{N}(\vec{r})$ is the state transition matrix for an individual object. It depends on the population $N$ and on the value $\vec{r}$ of the global memory. $K_{i, j}^{N}(\vec{r})$ is a transition probability from state $i$ to state $j$, therefore we assume that $K_{i, j}^{N}(\vec{r}) \geq 0$ and $\sum_{j=1}^{S} K_{i, j}^{N}(\vec{r})=1$. We further assume that

H For all $i, j$, for $N \rightarrow \infty, K_{i, j}^{N}(\vec{r})$ converges uniformly in $\vec{r}$ to some $K_{i, j}(\vec{r})$, which is a continuous function of $\vec{r}$.

Hypothesis $\mathbf{H}$ is true in particular if $K_{i, j}^{N}(\vec{r})$ does not depend on $N$ and is continuous in $\vec{r}$ (if so we write $K$ instead of $\left.K^{N}\right)$. Note that $\vec{R}^{N}(t)$ and $\vec{M}^{N}(t)$ are random vectors, but $\vec{R}^{N}(t)$ depends deterministically upon $\vec{M}^{N}(t)$.

Typically, the number of states for each object $S$ and the dimension of the memory $d$ is not large, in contrast the number of objects $N$ is very large; the number of possible states for the complete system is lower bounded by $S^{N}$, yet a larger number.

\subsection{Example without Memory}

A simple, special case of the model is when $\vec{R}^{N}(t)=$ $\vec{M}^{N}(t)$, i.e. the global memory is in fact the last value of 
the occupancy measure. In this case we say that the model is "without memory". Here $d=S$, the mapping $g()$ is reduced to $g(\vec{r}, \vec{m})=\vec{m}$, the state transition matrix is a function of $\vec{M}^{N}(t)$ and does not depend on $N$.

Consider for example a model of Robot Swarm found in [11]. We give here a simplified version, more realistic models are given in [11]. A set of $N$ robots travel across an area where there are $G_{0}$ grip sites. In each grip site there is a task to be performed; the task requires two robots and is performed in one time slot. The robot is either in search $(s)$ or grip $(g)$ state. A robot in state $s$ moves to state $g$ when it finds an empty grip site. A robot in state $g$ can be helped by some other robot, in which case it returns to state $s$; else, it may continue waiting, or gives up and returns to state $s$. See Figure 1 for the transition probabilities. This model

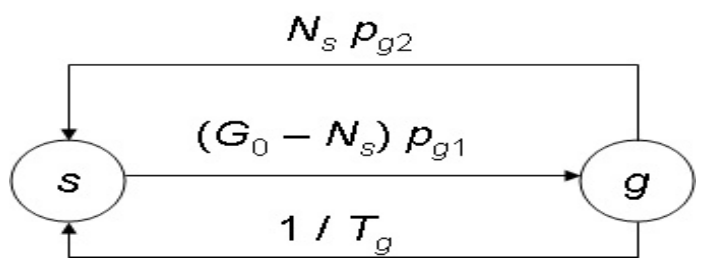

Figure 1. Finite State Machine for one robot, with transition probabilities; $N_{s}$ [resp. $N_{g}$ ] = number of robots in state $s$ [resp. $g$ ]; $G_{0}, p_{g_{1}}$, $p_{g_{2}}$ and $T_{g}$ are fixed parameters.

fits in our framework; an object is a robot, $\mathcal{E}=\{s, g\}$, $\vec{R}^{N}(t)=\vec{M}^{N}(t)=\frac{1}{N}\left(N_{s}(t), N_{g}(t)\right)$. To keep a discrete time setting meaningful, we need to assume that the values of $p_{g_{1}}, p_{g_{2}}$ and $G_{0}$ scale with $N$ according to $p_{g_{1}}=\frac{p_{1}}{N}$, $p_{g_{2}}=\frac{p_{2}}{N}$ and $G_{0}=g_{0} N$. We then have a model with transition matrix independent of $N: K_{s, g}\left(\vec{M}^{N}(t)\right)=\left(g_{0}-\right.$ $\left.M_{s}(t)\right) p_{1}, K_{g, s}\left(\vec{M}^{N}(t)\right)=M_{s}(t) p_{2}+\frac{1}{T_{g}}$. A deterministic approximation of this model is [11]:

$$
\begin{aligned}
N_{s}(t+1)= & N_{s}(t)\left(1-p_{g_{1}}\left(G_{0}-N_{g}(t)\right)\right) \\
& +N_{g}(t)\left(N_{s}(t) p_{g_{2}}+\frac{1}{T_{g}}\right)
\end{aligned}
$$

We see later (Equation (9)) how to derive this approximation in a systematic way, and show that it is indeed valid for a large number of robots.

\subsection{Example with Memory}

This model is for TCP connections over a RED/ECN Gateway. It was introduced and analyzed (including convergence to mean field) in [17]. The set of objects is the set

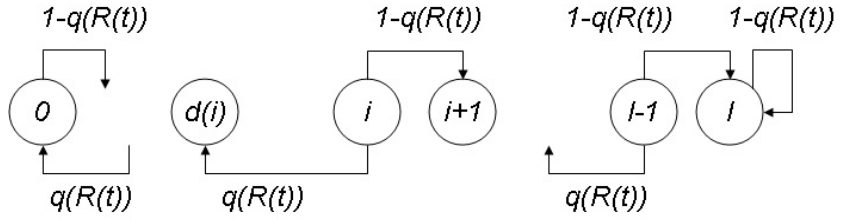

Figure 2. Finite State Machine for one TCP connection.

of $N$ TCP connections sharing a RED (random early detection) bottleneck. The state of an object is an index that defines its sending rate, i.e. when the state of a connection is $i$ the sending rate is $s_{i}$; there is a finite number of possible sending rates and $\mathcal{S}=\{0,1, \ldots, I\}$. The occupancy measure $\vec{M}^{N}(t)$ is the distribution of sending rates of all connections. Here $d=1$ and the memory $R^{N}(t)$ is the re-scaled queue size at the bottleneck, i.e. the buffer back$\log$ divided by $N$. It is assumed in [17] that the bottleneck capacity is $N C$, so that the update equation is:

$$
R^{N}(t+1)=\max \left(R^{N}(t)+S^{N}(t+1)-C, 0\right)
$$

where $S^{N}(t)$ is the per connection average sending rate at time $t$, i.e.

$$
S^{N}(t)=\sum_{i} s_{i} M_{i}^{N}(t) .
$$

The mapping $g()$ is thus defined by

$$
g(r, \vec{m})=\max \left(r+\sum_{i} s_{i} m_{i}-C, 0\right)
$$

The state of a connection is updated according to an increase/decrease rule. A TCP connection receives a negative feedback with a probability $q\left(R^{N}(t)\right)$ that depends on the re-scaled queue size $R^{N}(t)$. If no feedback is received, the rate is increased and the rate index goes from $i$ to $i+1$ (with $s_{i+1}>s_{i}$ ), except for $i=I$ in which case there is no increase. If a negative feedback is received, the rate is decreased and the rate index goes from $i$ to $d(i)$ (with $d(i)<i$ and consequently $s_{d(i)}<s_{i}$, except for $i=0$ where $d(0)=0$ ) (Figure 2). Here, too, the state transition matrix for an individual object does not depend on $N$; its non zero entries are:

$$
\begin{aligned}
K_{i, i+1}(r) & =(1-q(r)) 1_{\{i<I\}} \\
K_{I, I}(r) & =1-q(r) \\
K_{i, d(i)}(r) & =q(r)
\end{aligned}
$$

A mean field approximation of this system is 


$$
\begin{aligned}
M_{i}^{N}(t+1) & =\left(1-q\left(R^{N}(t)\right)\right) M_{i-1}^{N}(t) 1_{\{i>0\}} \\
& +q\left(R^{N}(t)\right) \sum_{j: d(j)=i} M_{j}^{N}(t) \text { for } i<I \\
M_{I}^{N}(t+1) & =\left(1-q\left(R^{N}(t)\right)\right)\left(M_{I-1}^{N}(t)+M_{I}^{N}(t)\right)
\end{aligned}
$$

where $R^{N}(t)$ is updated by Equation (4). It is a special case of the deterministic approximation in Equation (9).

\subsection{Heterogeneous Example}

The model of interacting objects can easily model a heterogeneous population of objects, by introducing object classes. We illustrate this on one example.

Consider the same RED/ECN gateway example, but assume now that TCP connections are of two classes: normal and aggressive. An object (i.e. a TCP connection) now has a state $(c, i)$, where $c$ denotes the class $(c=1$ [resp. 2] for the normal [resp. aggressive] class) and $i$ is, as before, the index of the sending rate. When a normal [resp. aggressive] TCP connection receives a feedback, its rate index is decreased from $i$ to $d_{1}(i)$ [resp. $d_{2}(i)$ ], with $d_{1}(i) \leq d_{2}(i)$. The rest is as in the previous example.

Assume first that there is no class change. Then the state transition matrix is independent of $N$ :

$$
\begin{aligned}
K_{c, i, c, i+1} & =(1-q(r)) 1_{\{i<I\}} \\
K_{c, I, c, I} & =1-q(r) \\
K_{c, i, c, d_{c}(i)}(r) & =q(r)
\end{aligned}
$$

for $c=1,2$ and $i=0, \ldots, I$ and $K_{c, i, c^{\prime}, i^{\prime}}=0$ in all other cases. A deterministic approximation can be derived for $M_{c, i}^{N}(t)$ similar to Equation (5). Let $p_{c}$ be the proportion of class $c$ TCP connections; $p_{c}$ is independent of time since we assume there is no class change. The proportion of class $c$ connections in state $i$ at time $t$ is then $\frac{1}{p_{c}} M_{c, i}^{N}(t)$.

Assume next that a connection may change class, and let $q_{c, c^{\prime}}(R(t))$ be the probability that a connection that is in class $c$ at time $t$ switches to class $c^{\prime}$ for the next time slot. The state transition matrix is now given by

$$
\begin{aligned}
K_{c, i, c^{\prime}, i+1} & =(1-q(r)) 1_{\{i<I\}} q_{c, c^{\prime}}(r) \\
K_{c, I, c^{\prime}, I} & =(1-q(r)) q_{c, c^{\prime}}(r) \\
K_{c, i, c^{\prime}, d_{c}(i)}(r) & =q(r) q_{c, c^{\prime}}(r)
\end{aligned}
$$

for $c=1,2$ and $i=0, \ldots, I$ and $K_{c, i, c^{\prime}, i^{\prime}}=0$ in all other cases. The proportion of class $c$ connections at time $t$ is now $p_{c}(t)=\sum_{i} M_{c, i}^{N}(t)$ and the proportion of class $c$ connections that are in state $i$ is $\frac{1}{p_{c}(t)} M_{c, i}^{N}(t)$.

In Section 6 we analyze in detail a real size application (reputation determination), which uses models with and without memory, and where the transition matrix depends on $N$.

\section{Convergence to Mean Field}

\subsection{Convergence Result}

The occupancy measure $\vec{M}^{N}(t)$ and thus the global memory $\vec{R}^{N}(t)$ are random: for two different simulation runs, one obtains different values. The following theorem says that, as $N \rightarrow \infty$, randomness goes away and one can replace $\vec{M}^{N}(t)$ and $\vec{R}^{N}(t)$ by deterministic approximations $\vec{\mu}(t)$ and $\vec{\rho}(t)$. A weaker form of this theorem (convergence in probability instead of almost sure) was first introduced, in the case without memory, and proven in [3]. A similar, but less general, theorem was proven in [17] for the specific case, with memory, of the example in Section 3.3.

Theorem 4.1 (Convergence to Mean Field) Assume that the initial occupancy measure $\vec{M}^{N}(0)$ and memory $\vec{R}^{N}(0)$ converge almost surely to deterministic limits $\vec{\mu}(0)$ and $\vec{\rho}(0)$ respectively. Define $\vec{\mu}(t)$ and $\vec{\rho}(t)$ iteratively by their initial values $\vec{\mu}(0), \vec{\rho}(0)$ and for $t \geq 0$ :

$$
\begin{aligned}
\vec{\mu}(t+1) & =\vec{\mu}(t) \cdot K(\vec{\rho}(t)) \\
\vec{\rho}(t+1) & =g(\vec{\rho}(t), \vec{\mu}(t+1))
\end{aligned}
$$

Then for any fixed time $t$, almost surely:

$$
\lim _{N \rightarrow \infty} \vec{M}^{N}(t)=\vec{\mu}(t) \text { and } \lim _{N \rightarrow \infty} \vec{R}^{N}(t)=\vec{\rho}(t) .
$$

We give a proof in appendix.

Comments. Equation (6) means that for any state $i$ :

$$
\mu_{i}(t+1)=\sum_{j=1}^{S} \mu_{j}(t) K_{j, i}(\vec{\rho}(t))
$$

The conditions $\vec{M}^{N}(0) \rightarrow \vec{\mu}(0), \vec{R}^{N}(0) \rightarrow \vec{\rho}(0)$ are true in particular if $\vec{M}^{N}(0)=\vec{\mu}(0)$ and $\vec{R}^{N}(0)=\vec{\rho}(0)$, which is often the case in practice.

The theorem implies that, over any finite time horizon the global memory and the occupancy measure (i.e. the number of objects in any given state) can be approximated, for a large population size $N$, by the dynamical system defined by Equations (6) and (7). Note that the theorem says nothing about the limit when time (rather than population size) goes to $\infty$.

The time dependent deterministic vector $\vec{\mu}(t)$ is called the mean field limit of the occupancy measure $\vec{M}^{N}(t)$.

\subsection{Deterministic Approximation}

We can use the convergence result to justify the common practice that consists in approximating the stochastic 
system by a deterministic one. Let $N_{i}(t)$ be the number of objects that are in state $i$ at time $t$, i.e. $N_{i}(t)=N M_{i}^{N}(t)$. Since $N$ is fixed, knowing $\vec{N}(t)$ or $\vec{M}^{N}(t)$ is one and the same. In the rest of this subsection, we consider that $N$ is fixed and drop superscript $N$.

A common approximation consists in assuming that $N_{i}(t)$ is deterministic and:

$$
\begin{aligned}
N_{i}(t+1)= & N_{i}(t)\left(1-\sum_{j \neq i} K_{i, j}(\vec{\rho}(t))\right) \\
& +\sum_{j \neq i} N_{j}(t) K_{i, j}(\vec{\rho}(t))
\end{aligned}
$$

with $\vec{\rho}(t)=g(\vec{\rho}(t-1), \vec{N}(t) / N)$; see for example Equation (3).

It is immediate that Equation (9) is a re-writing of Equation (8). Thus the deterministic limit is asymptotically valid for a large population of objects.

\section{Fast Simulation}

We extend Theorem 4.1 to a more detailed result, which can be used to perform accelerated simulation. The idea is as follows. Assume we want to simulate the evolution of a few specific objects, perhaps because we want to evaluate the time it takes to reach some specific state. We can do this in an accelerated way by replacing the true occupation measure $\vec{M}^{N}(t)$ and the global memory $\vec{R}^{N}(t)$ (the exact evaluation of which requires simulating $N$ objects) by their deterministic approximation $\vec{\mu}(t)$ and $\vec{\rho}(t)$ (obtained from the dynamical system in Equations (6) and (7)). The difference is that now we need to do a stochastic simulation only for the objects we are interested in. One may even consider doing analytical computations, since for one object the dimension of the Markov chain is $S$, a small number. The main result in this section is that, for large $N$, this is a valid approximation.

To make this precise, call $\mathcal{P}^{N}(i, \vec{r}, u)$ a program that returns a sample of the next state for an object that is in state $i$ when the global memory is $\vec{r}$, according to Equation (2). The argument $u$ is a random number in the interval $(0,1)$, typically provided by a pseudo-random number generator. More precisely, $\mathcal{P}^{N}(i, \vec{r}, u)=j$ if and only if

$$
\sum_{j^{\prime}=1}^{j-1} K_{i, j^{\prime}}^{N}(\vec{r})<u \leq \sum_{j^{\prime}=1}^{j} K_{i, j^{\prime}}^{N}(\vec{r})
$$

where we use the convention that $\sum_{j^{\prime}=1}^{0} K_{i, j^{\prime}}^{N}(\vec{r})=0$.

An exact simulation of the complete system can be described as follows (Algorithm 1). We are given a time horizon $t$, a total number of objects $N$, the collection $\mathbf{X}^{N}(0)=$
$\left(X_{1}^{N}(0), \ldots, X_{N}^{N}(0)\right)$ of initial values for all objects, and the initial value of global memory $\vec{R}^{N}(0)$. The program outputs $\mathbf{X}^{N}(1: t)$, a sample of the states of all objects at times $s=1$ to $t$.

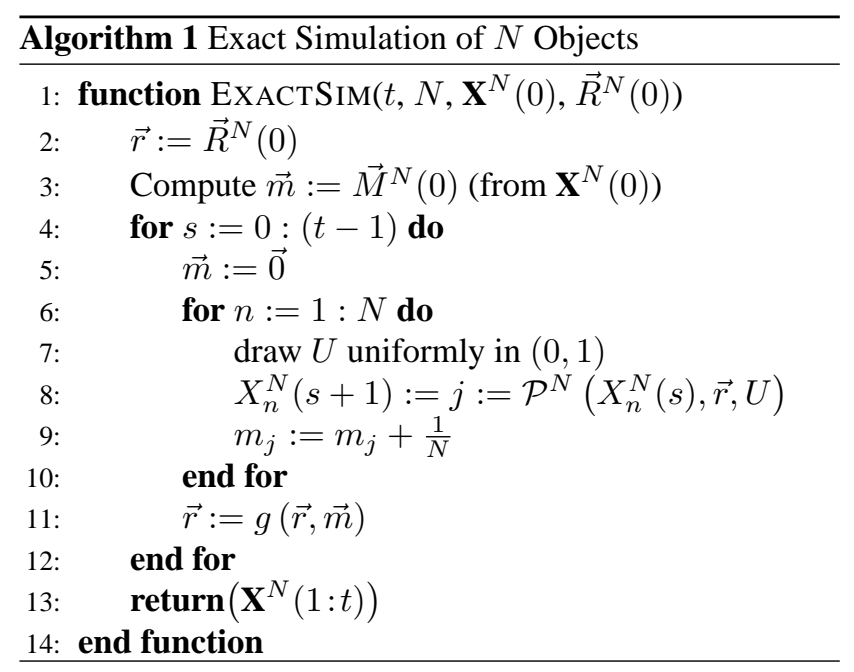

The fast simulation is defined as follows (Algorithm 2). Define $\mathcal{P}$ similar to $\mathcal{P}^{N}$ but with $K^{N}$ replaced by $K$. We are given a time horizon $t$, the initial state $y(0)$ of, say, object number $n=1$, the initial occupancy measure $\vec{\mu}(0)$ and the initial value of global memory $\vec{\rho}(0)$. The program outputs $Y_{1}(s)$, a sample of the state of object 1 at times $s=1$ to $t$.

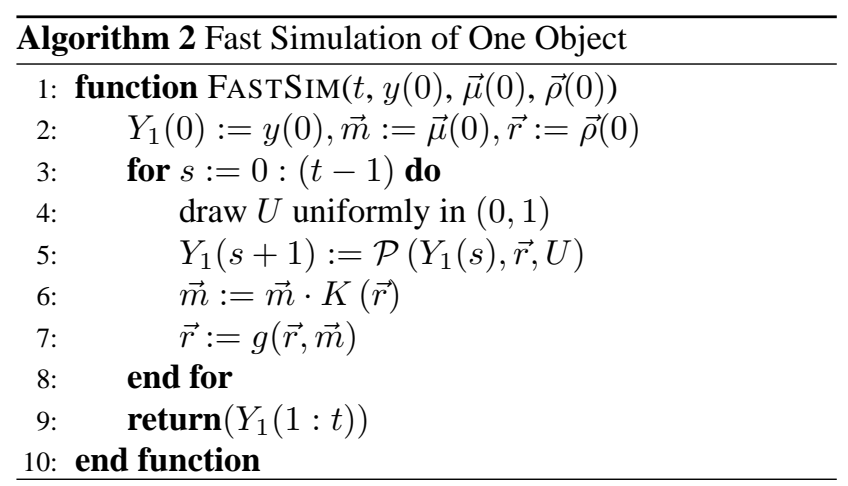

Note that in the case without memory, the argument $\vec{\rho}(0)$ need not be specified.

Theorem 5.1 Assume that we perform a collection of simulations using the above algorithms, indexed by $N$, such that:

- for all $N$ the initial state of object 1 is $y(0)$

- the initial occupancy measure $\vec{M}^{N}(0)$ and memory $\vec{R}^{N}(0)$ converge almost surely to deterministic limits $\vec{\mu}(0)$ and $\vec{\rho}(0)$ respectively 
- the random numbers used by FASTSIM are the same as those used by EXACTSIM when computing $X_{1}^{N}(s)$.

Then, almost surely, for $N$ large enough, $X_{1}^{N}(s)=Y_{1}(s)$ for $s=1$ to $t$.

The proof is given in appendix. We give a detailed application example in the next section.

We can apply Theorem 5.1 many times in parallel and simulate not just one object but $n_{0}$ of them, perhaps each with different initial conditions (see Figure 3, bottom, for an example with $n_{0}=3$ ). It follows from the theorem that the simulations can be done independently, since the random numbers used by FASTSIM are all drawn independently. Thus, as long as we are interested in a finite number of objects over a finite horizon and when the total number of objects $N$ is large, the evolutions of $n_{0}$ objects are independent of each other (but are controlled by the value of the global memory).

If, in addition, the initial states of the $n_{0}$ objects are drawn independent of each other and from a common distribution, then, asymptotically in $N$, the $n_{0}$ objects are independent identically distributed at all times. This is called in the literature "propagation of chaos". Note that in the examples of the next section, this does not hold as the initial states of the objects of interest are not drawn from the same distribution.

\section{Application to Reputation determination}

In this section we illustrate how the mean field convergence and simulation results can be applied to the analysis of a reputation system.

\subsection{Reputation determination}

The setting is a simplified version of a reputation system in [6]. A number of peers keep a reputation rating of a single subject (for example a TV show or a restaurant); the rating maintained by peer $n$ is a number $Z_{n}$ in the interval $[0,1]$. At every time step, peer $n$ either makes a direct or an indirect observation. The reputation system attempts to model the effects of forgetting and confirmation bias (explained next).

A direct observation is assumed to be positive or negative. The probability that a direct observation is positive is $\theta$, interpreted as the true value of the subject. When peer $n$ makes a positive direct observation at time $t+1$, he sets his rating to

$$
Z_{n}(t+1)=w Z_{n}(t)+(1-w)
$$

and if the observation is negative, to:

$$
Z_{n}(t+1)=w Z_{n}(t)
$$

In these equations, $w$ is a constant close to 1 , which represents the decay of stored information ("forgetting").

An indirect observation consists in observing the reputation rating maintained by some arbitrary peer. We assume that there are honest peers and liars. If the sampled peer is honest, the rating is her reputation value. If he is a liar, we assume first ("Strategy 1: Non Sophisticated Liar" model) that the sampled rating is 0 , in other words, liars attempt to propagate the worst possible reputation. Later in the paper we will consider more sophisticated strategies. A peer, say $n$, who observes a reputation value $z$ makes a deviation test. If the sampled rating $z$ differs by more than $\Delta$, the observation is discarded. Otherwise, it is updated in a form similar to direct observation. The deviation test represents what is called in social sciences the "confirmation bias". Thus the update for an indirect observation $z$ is

$$
\begin{aligned}
& Z_{n}(t+1)=Z_{n}(t) \text { if }\left|z-Z_{n}(t)\right|>\Delta \\
& Z_{n}(t+1)=w Z_{n}(t)+(1-w) z \text { otherwise }
\end{aligned}
$$

We assume that an observation is direct with probability $p$, indirect with a liar with probability $q$, and indirect with an honest peer with probability $r$. The probability that an object makes an observation in one time slot is thus $p+q+r$. In the rest of the paper, we assume, to simplify, that $p+q+r=1$.

This system was introduced and studied in [14], with generalizations analysed in [13] and [15]. The approach there was to derive an ordinary differential equation from the stochastic process by averaging the dynamics and passing to a fast-time scaling limit. (That is, scaling time so that events occur more frequently, i.e. users make observations at a higher rate, but at the same time the impact of each observation is reduced by the same factor.) From this, its fixed points reputation ratings were obtained. It has not been rigorously shown that the set of fixed point solutions of the stochastic process is contained in the set of fixed point solutions of differential equation, but results were verified by means of simulation of up to several hundred peers as well as direct computation of the distribution.

It was found that, depending on the parameters, there are up to two attracting reputation ratings. One of them is the true value $\theta$, the other is $\theta p /(p+q)$. If $\Delta<\frac{p \theta}{p+q}$ only the former $(\theta)$ exists; if $\frac{p \theta}{p+q}<\Delta<\theta$ both exist and if $\Delta<\theta$, only the latter. For example, when $\theta>\Delta$, in order to have an impact, the number of liars in the network needs to exceed a certain threshold, i.e. one must have $q>$ $p\left(\frac{\theta}{\Delta}-1\right)$. Full details and derivations can be found in [12]. We will see next that this heuristic method is confirmed by 


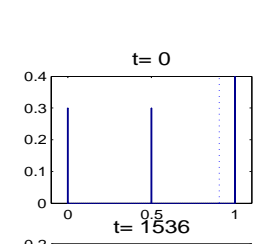

$$
N=100
$$
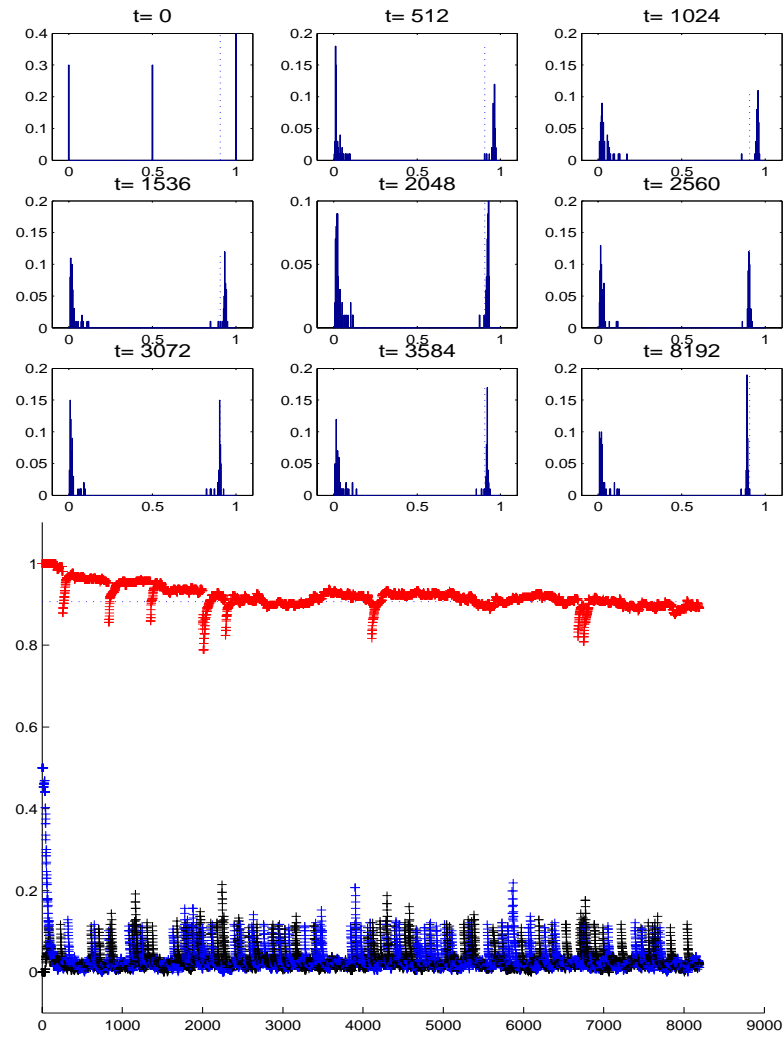
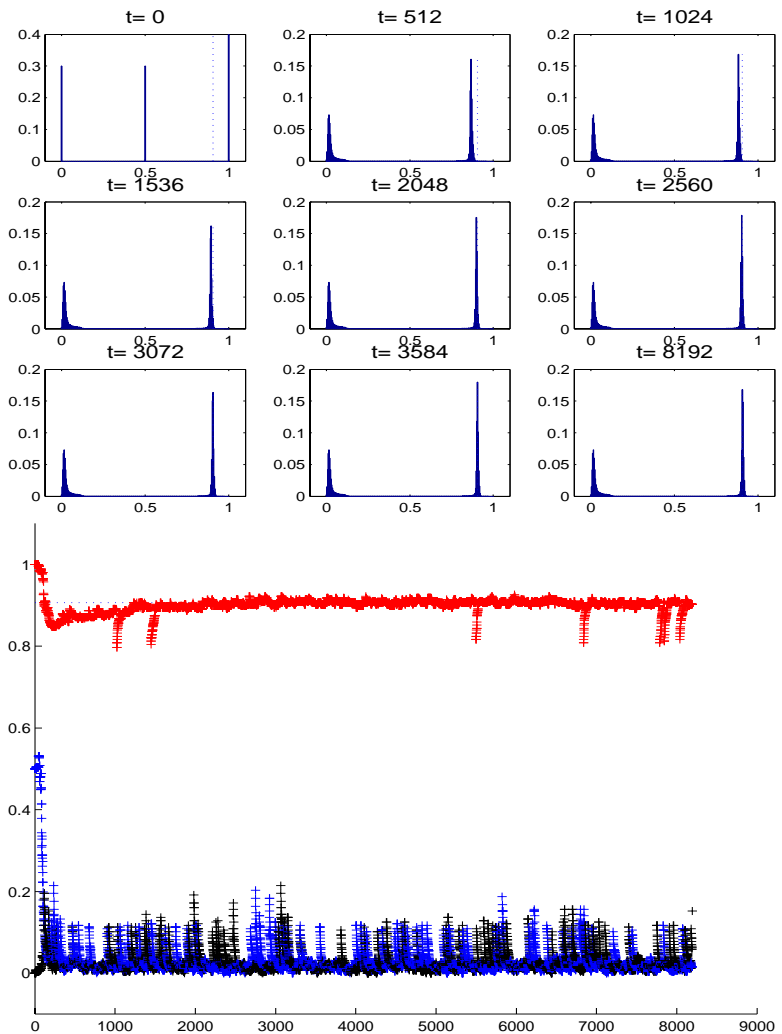

$N=\infty$
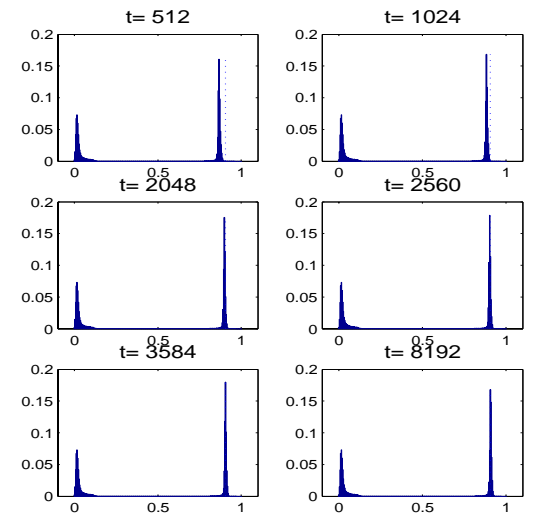

\section{(1)}

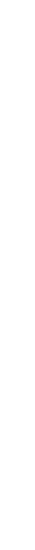

Figure 3. Simulation results for the setting in Section 6.4. Top Panels: Histogram of reputation ratings, with one panel per time instant, for selected times from $t=0$ to $t=8192$, "many liars" case; $x$-axis is the rating value on a $0-1$ scale, $y$-axis: fraction of peers that have this value. Bottom: sample paths for three different honest users, starting with initial reputation rating $\in\{0,0.5,1\} ; x$ axis is time, $y$-axis is rating value on a $0-1$ scale. Left: $N=100$ honest users; right: $N=\infty$. Dashed vertical or horizontal lines are at the true value $\theta$. There are two attracting values for the ratings, the true value $\theta=$ and a false value close to 0 .

the asymptotic results in this paper, but we will also see how we can get a more accurate picture.

\subsection{Discretization}

The ratings, as originally defined, are floating point numbers. In order to apply the modelling approach in this paper, we need to transform the problem so that the set of reputation rating values is discrete. We describe this in this section.

We replace the original ratings $Z_{n}(t) \in[0,1]$ by integer valued ratings $X_{n}(t) \in\left\{0, \ldots, 2^{L}\right\}$, for some fixed value of $L$. In the rest of the paper we take $L=8$, so that there are 257 possible reputation rating values. Of course, we re-scale the updates in Equations (11) to (14) appropri- ately. However, in doing so, the results may not be integers, even if all state variables are, because of the decay factor $w$ (which is not re-scaled). A simple fix would consist in rounding the right-hand sides of these equations to the nearest integers, but this may not be very accurate because the decay factor $w$ is typically close to 1 . Indeed, with this form of discretization, many small updates may simply result in no change at all, if each update is small compared to the discretization step.

To avoid this problem, we use probabilistic rounding. We use the function $\operatorname{RANDROUND}(x)$, defined for $x \in$ $\mathbb{R}$, which returns a random number equal to one of the two integers nearest to $x$, and is in expectation equal to $x$. Specifically, $\operatorname{RANDROUND}(x)=x$ if $x \in \mathbb{Z}$, else $\operatorname{RANDROUND}(x)=\lceil x\rceil$ with probability frac $(x)$ and $\lfloor x\rfloor$ with probability $1-\operatorname{frac}(x)$. We used the following nota- 
tion: $\lfloor x\rfloor$ is the largest integer $\leq x,\lceil x\rceil$ the smallest integer $\geq x$ and $\operatorname{frac}(x)=x-\lfloor x\rfloor$. Note that, for $x$ non integer, $\lfloor x\rfloor<x<\lceil x\rceil=\lfloor x\rfloor+1$ and, for $x$ integer, $\lfloor x\rfloor=x=$ $\lceil x\rceil$. Further, for any $x \in \mathbb{R}, \mathbb{E}(\operatorname{RANDROUND}(x))=x$.

Equations (11) to (14) are replaced by the following. In case of a positive direct observation:

$$
X_{n}(t+1)=w X_{n}(t)+2^{L}(1-w),
$$

and in case of a negative direct observation:

$$
X_{n}(t+1)=w X_{n}(t)
$$

In case of indirect observation $x$ such that $\left|x-X_{n}(t)\right| \leq$ $\Delta 2^{L}$

$$
X_{n}(t+1)=w X_{n}(t)+(1-w) x
$$

otherwise

$$
X_{n}(t+1)=X_{n}(t)
$$

\subsection{Formulation as a System of Interact- ing Objects}

We first consider strategy 1 . We model only the ratings of honest peers. We can cast the reputation system in the framework of Section 3, as follows. The $N$ objects are the reputation ratings of the $N$ honest peers. The state of an object is the rating value, thus $\mathcal{E}=\left\{0,1,2, \ldots, 2^{L}\right\}$. The next state is determined only by the current state and the distribution of states of all honest peers. Thus, this fits in our modelling framework, in fact we have a model without memory. We will see later that for strategy 2 we have a model with memory.

Specifically, we can express the state update equation by using Equations (15) to (18). The probability that an indirect observation by peer $n$, sampled from an honest peer, is $k$ is equal to

$$
\frac{N M_{k}^{N}(t)-1_{\left\{k=X_{n}(t)\right\}}}{N-1}
$$

because a peer does not meet with herself. Thus

$$
X_{n}(t+1)=X_{n}(t)+\operatorname{RANDROUND}((1-w) U)
$$

with $U=$

$$
\begin{aligned}
& \left(2^{L}-X_{n}(t)\right) \text { with probability } p \theta \\
& -X_{n}(t) \text { with probability } p(1-\theta) \\
& -X_{n}(t) 1_{\left\{X_{n} \leq \Delta 2^{L}\right\}} \text { with probability } q \\
& \left(k-X_{n}(t)\right) 1_{\left\{\left|k-X_{n}(t)\right| \leq \Delta 2^{L}\right\}}(t)-1_{\left\{k=X_{n}(t)\right\}} \\
& \quad \text { w. p. }(1-p-q) \frac{N M_{k}^{N}(1}{N-1}
\end{aligned}
$$

The limiting state transition matrix $K_{i, j}(\vec{m})$ can be derived by letting $N \rightarrow \infty$, which amounts to replacing the term
$\frac{N M_{k}^{N}(t)-1_{\left\{k=X_{n}(t)\right\}}}{N-1}$ in Equation (19) by $M_{k}^{N}(t)$. The rest follows in a straightforward but tedious way. First define $K^{*}$ by

$$
K_{i, j}^{*}(\vec{m})=K_{i, j}^{1}+K_{i, j}^{2}+\sum_{k=0}^{2^{L}} K_{i, j}^{3, k}(\vec{m})
$$

with

$$
\begin{aligned}
& K_{i, j}^{1}=p \theta \times \\
& \left(\pi_{i}^{1} 1_{\left\{j=i+\left\lceil(1-w)\left(2^{L}-i\right)\right\rceil\right\}}+\left(1-\pi_{i}^{1}\right) 1_{\left\{j=i+\left\lfloor(1-w)\left(2^{L}-i\right)\right\rfloor\right\}}\right) \\
& K_{i, j}^{2}=\left(p(1-\theta)+q 1_{\left\{0 \leq i \leq \Delta 2^{L}\right\}}\right) \times \\
& \left(\pi_{i}^{2} 1_{\{j=i+\lceil-(1-w) i\rceil\}}+\left(1-\pi_{i}^{2}\right) 1_{\{j=i+\lfloor-(1-w) i\rfloor\}}\right) \\
& K_{i, j}^{3, k}(\vec{m})=\left((1-p-q) m_{k} 1_{\left\{|k-i| \leq \Delta 2^{L}\right\}}\right) \times \\
& \left(\pi_{i}^{3, k} 1_{\{j=1+\lceil(1-w)(k-i)\rceil\}}+\left(1-\pi_{i}^{3, k}\right) 1_{\{j=1+\lfloor(1-w)(k-i)\rfloor\}}\right) \\
& \pi_{i}^{1}=\operatorname{frac}\left((1-w)\left(2^{L}-i\right)\right) \\
& \pi_{i}^{2}=\operatorname{frac}(-(1-w) i) \\
& \pi_{i}^{3, k}=\operatorname{frac}((1-w)(k-i))
\end{aligned}
$$

and finally let $K_{i, j}(\vec{m})=K_{i, j}^{*}(\vec{m})$ for $i \neq j$ and $K_{i, i}(\vec{m})=1-\sum_{j} K_{i, j}^{*}(\vec{m})$.

We do not write in detail the $N$-dependent value $K_{i, j}^{N}(\vec{m})$ but it can easily be seen that it differs from $K_{i, j}(\vec{m})$ by at most $\frac{2}{N-1}$ (due to the last line in Equation (19)). It follows that $K_{i, j}^{N}(\vec{m})$ converges uniformly in $\vec{m}$ to $K_{i, j}(\vec{m})$, and the limit is continuously in $\vec{m}$ (in fact, linear), as required by hypothesis $\mathbf{H}$. Thus, for large $N$, we have convergence towards the mean field. We illustrate this in the next section.

\subsection{Simulation Results}

We can apply Theorem 4.1 and compute the distribution of reputations ratings as a function of time. We used the following parameters. The reputation value is coded on $L=8$ bits. The deviation test threshold is $\Delta=0.40$ and the decay factor is $w=0.9$. The true value of the subject is $\theta=0.90625$. At every time slot, an honest peer makes a direct experience with probability $p=0.01$, learns indirect information from a liar with probability $q=0.01$ ("few liars" case) or 0.3 ("many liars" case), and from an honest peer with probability $1-p-q$. The initial occupancy measure $\vec{\mu}(0)$ is such that a fraction $\alpha=0.3$ of honest peers initially believes that the subject is bad $\left(Z_{n}(0)=0\right)$, a fraction $\beta \in\{0.3,0.38,0.45\}$ of honest peers is undecided $\left(Z_{n}=0.5\right)$ and the rest believes that the subject is good $\left(Z_{n}(0)=1\right)$. 

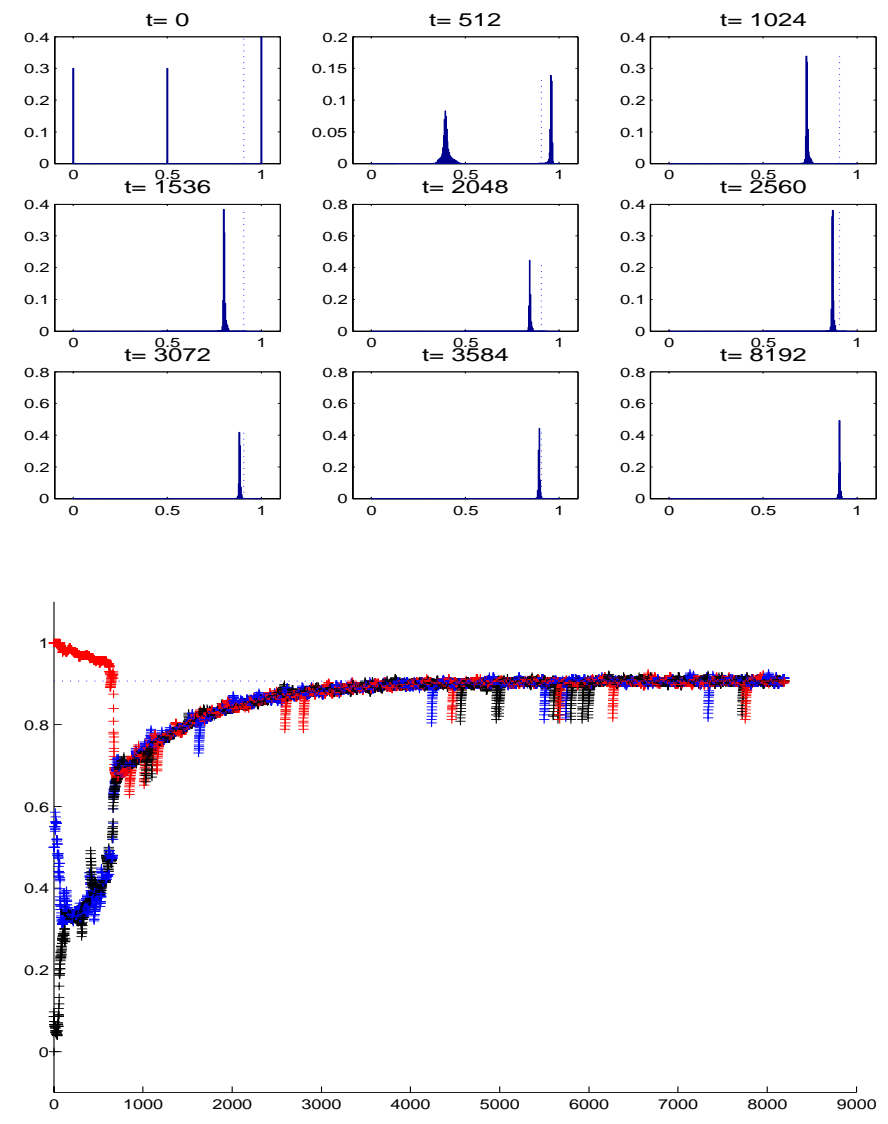

Figure 4. "Few liars" case, $N=\infty$. There is only one attracting value for the ratings, the true value $\theta$.

Figure 3 shows the evolution of the occupancy measure and samples of reputation records for 3 typical peers with different initial values ( $\beta=0.3$, "many liars" case), both for a system with $N=100$ honest peers (obtained with the EXACTSIM algorithm) and for the large $N$ asymptotics (by application of Theorem 4.1 and Algorithm 2). We see that the asymptotic method agrees with the $N=100$ case.

The approximate analysis in $[14,13,15]$ predicts that for the "many liars" case, there are two attracting reputation values, one of them is the true value $\theta$, the other is $\theta p /(p+q)$. This is confirmed by Figure 3, where we see that the distribution of reputation records converges towards a distribution concentrated around the two predicted attracting reputation ratings. In the "few liars case", the approximate analysis predicts that only the true value is remains, which is indeed confirmed by Figure 4 (shown for $\beta=0.3$ ).

Not only does the mean field approach justify the approximate analysis, in the former case ("many liars") it also
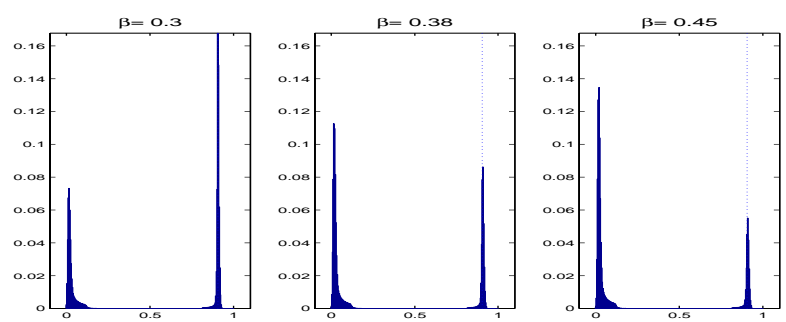

Figure 5. Stationary values of the occupancy measure for $N$ large and for different initial conditions ("many liars" case). The initial number of peers with rating $=0.5$ is $\beta=0.3$, 0.38 or 0.45 . The final distribution depends on the initial conditions

gives a more accurate result: we see that there are indeed two concentration of the occupancy measures around the two attracting reputation values, but we see also that the masses depend on the initial values (Figure 5).

We can also recover the attracting reputation values computed in $[14,13,15]$ as follows. Assume that the occupancy measure converges to a mass concentrated at a single value $i^{*}$, i.e. $\vec{\mu}(t)=\delta_{i *}$, where $\delta_{i^{*}}$ is a row vector with $S$ columns such that $\left(\delta_{i^{*}}\right)_{i}=0$ for $i \neq i^{*}$ and $\left(\delta_{i *}\right)_{i}=1$. By application of Equation (6), one founds $\delta_{i^{*}}=\delta_{i^{*}} K\left(\delta_{i^{*}}\right)$, which gives an equation for $i^{*}$. Solving the equation gives the two attracting reputation values discussed above.

\subsection{Other Liar Strategies}

In this section we show how we can model other liar strategies.

\section{Strategy 2: Inferring Liar}

A liar tries to guess the rating of the honest peer she meets, by assuming the next meeting will be similar to some previous reputation rating. A liar says her value is ${ }^{1} x=$ $\left(Y-\Delta^{\prime}\right)^{+}$, where $Y$ is sampled from the occupancy measure at the previous time slot and where $\Delta^{\prime} \approx 0.75 \Delta$. The coefficient 0.75 is a safety margin. We assume for simplicity (and without much loss of generality) that $\Delta^{\prime}$ is chosen such that $(1-w) \Delta^{\prime} 2^{L} \in \mathbb{N}$.

We continue to model honest peers only, but now use now a model with memory, since liars base their value on the occupancy measure at the previous time slot. We take

$$
\vec{R}^{N}(t)=\left(\vec{M}^{N}(t), \vec{M}^{N}(t-1)\right)
$$

\footnotetext{
${ }^{1}$ We use the notation $(z)^{+}=\max (z, 0)$.
} 
so that the mapping $g()$ is defined by

$$
g\left(\vec{r}, \vec{m}_{\text {new }}\right)=\left(\vec{m}_{\text {new }}, \vec{m}\right)
$$

with $\vec{r}=\left(\vec{m}, \vec{m}^{\prime}\right)$. The formulation as a system of interacting objects is the same as before, except that we replace the limit for large $N$ of Equation (19) by $U=$

$$
\begin{aligned}
& \left(2^{L}-X_{n}(t)\right) \text { with probability } p \theta \\
& -X_{n}(t) \text { with probability } p(1-\theta) \\
& \left(\left(k^{\prime}-\Delta^{\prime} 2^{L}\right)^{+}-X_{n}(t)\right) 1_{\left\{\left|\left(k^{\prime}-\Delta^{\prime} 2^{L}\right)^{+}-X_{n}(t)\right| \leq \Delta 2^{L}\right\}} \\
& \quad \text { with probability } q M_{k^{\prime}}^{N}(t-1) \\
& \left(k-X_{n}(t)\right) 1_{\left\{\left|k-X_{n}(t)\right| \leq \Delta 2^{L}\right\}} \\
& \quad \text { with probability }(1-p-q) M_{k}^{N}(t)
\end{aligned}
$$

The fast simulation is obtained by replacing Equation (20) by

$$
K_{i, j}^{*}\left(\vec{m}, \vec{m}^{\prime}\right)=K_{i, j}^{1}+K_{i, j}^{2}+\sum_{k=0}^{2^{L}} K_{i, j}^{3, k}(\vec{m})+\sum_{k^{\prime}=0}^{2^{L}} K_{i, j}^{4, k^{\prime}}\left(\vec{m}^{\prime}\right)
$$

with $K_{i, j}^{1}, K_{i, j}^{3, k}, \pi_{i}^{1}, \pi_{i}^{2}, \pi_{i, j}^{3}$ as with Strategy 1 and

$$
\begin{aligned}
& K_{i, j}^{2}=(p(1-\theta)) \times \\
& \quad\left(\pi_{i}^{2} 1_{\{j=i+\lceil-(1-w) i\rceil\}}+\left(1-\pi_{i}^{2}\right) 1_{\{j=i+\lfloor-(1-w) i\rfloor\}}\right) \\
& K_{i, j}^{4, k^{\prime}}\left(\vec{m}^{\prime}\right)=\left(q m_{k^{\prime}}^{\prime} 1_{\left\{\mid\left(k^{\prime}-\Delta^{\prime} 2^{L}\right)^{\left.+-i \mid \leq \Delta 2^{L}\right\}}\right) \times}\right) \times \\
& \quad\left(\pi_{i}^{4, k^{\prime}} 1_{\left\{j=1+\left\lceil(1-w)\left(\left(k^{\prime}-\Delta^{\prime} 2^{L}\right)^{+-i}\right)\right\rceil\right\}}+\right. \\
& \left.\quad\left(1-\pi_{i}^{4, k^{\prime}}\right) 1_{\left\{j=1+\left\lfloor(1-w)\left(\left(k^{\prime}-\Delta^{\prime} 2^{L}\right)+-i\right)\right\rfloor\right\}}\right) \\
& \pi_{i}^{4, k^{\prime}}=\operatorname{frac}\left((1-w)\left(\left(k^{\prime}-\Delta^{\prime} 2^{L}\right)^{+}-i\right)\right)
\end{aligned}
$$

Here, too, the matrix $K$ depends continuously on $\vec{r}=$ $\left(\vec{m}, \vec{m}^{\prime}\right)$, so we can apply the convergence results. See Figure 6 and Figure 7 for some numerical examples.

\section{Strategy 3: Side Information}

This is the extreme case where a liar knows the rating $X$ of the honest peer he meets. The liar pretends that his value is $x=\left(X-\Delta^{\prime}, 0\right)^{+}$, with $\Delta^{\prime}$ as in Strategy 2. This may not be realistic, but serves as an upper bound on what liars can achieve.

This can also be modelled as a system of interacting objects; replace Equation (21) by $U=$

$$
\begin{aligned}
& \left(2^{L}-X_{n}(t)\right) \text { with probability } p \theta \\
& -X_{n}(t) \text { with probability } p(1-\theta) \\
& -\min \left(X_{n}(t), \Delta^{\prime} 2^{L}\right) \text { with probability } q \\
& \left(k-X_{n}(t)\right) 1_{\left\{\left|k-X_{n}(t)\right| \leq \Delta 2^{L}\right\}} \\
& \quad \text { with probability }(1-p-q) M_{k}^{N}(t)
\end{aligned}
$$
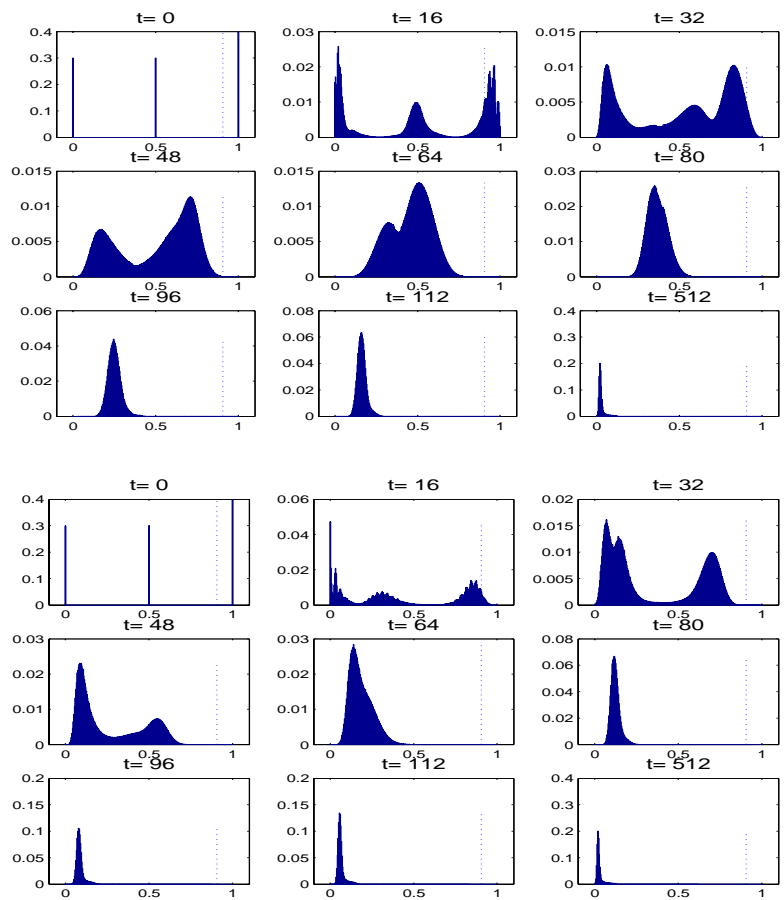

Figure 6. Different liar strategies. Top: Strategy 2 (Infer Peer Distribution); bottom: Strategy 3 (Side Information). Parameters are the same as Figure 3 but time is from 0 to 512 time units. All reputation ratings are quickly coming close to 0 .

The fast simulation is obtained by replacing Equation (20) by

$$
K_{i, j}^{*}(\vec{m})=K_{i, j}^{1}+K_{i, j}^{2}+\sum_{k=0}^{2^{L}} K_{i, j}^{3, k}(\vec{m})+K_{i, j}^{4}
$$

with $K_{i, j}^{1}, K_{i, j}^{3, k}, \pi_{i}^{1}, \pi_{i}^{2}, \pi_{i, j}^{3}$ as with strategy 1 and

$$
\begin{aligned}
& K_{i, j}^{2}=\left(p(1-\theta)+q 1_{\left\{i \leq \Delta^{\prime} 2^{L}\right\}}\right) \times \\
& \quad\left(\pi_{i}^{2} 1_{\{j=i+\lceil-(1-w) i\rceil\}}+\left(1-\pi_{i}^{2}\right) 1_{\{j=i+\lfloor-(1-w) i\rfloor\}}\right) \\
& K_{i, j}^{4, k^{\prime}}=1_{\left\{i>\Delta^{\prime} 2^{L}\right\}} q 1_{\left\{j=i-(1-w) \Delta^{\prime} 2^{L}\right\}}
\end{aligned}
$$

On Figure 6 we see that the mean field converges very much in the same way for strategies 2 and 3, but Figure 7 shows that there is a difference in what happens to a honest peer after the occupancy measure has converged. This nicely illustrates the importance of being able not only to approximate the occupancy measure by Theorem 4.1, but also to simulate the state of individual objects as per Theorem 5.1. 

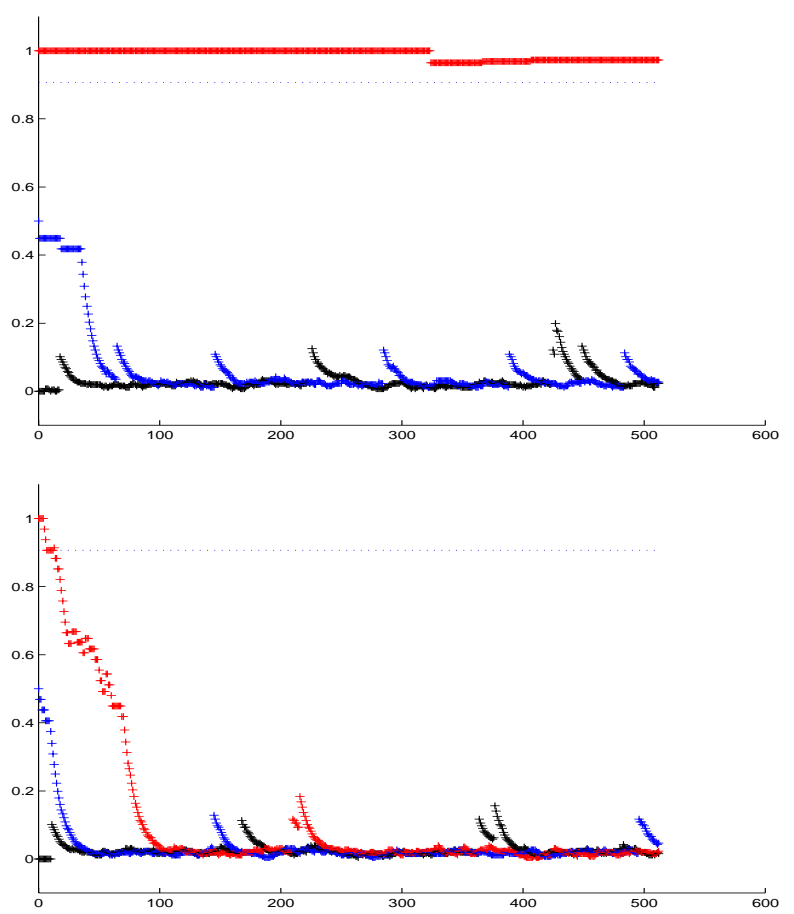

Figure 7. Three honest peers when liars use strategy 2 (top) or strategy 3 (bottom), after the occupancy measure has converged to the final values in Figure 6. Strategy 2 (unlike strategy 3 ) fails to deceive the honest peer, who is protected by the deviation test.

\section{Conclusion}

We have given a generic result that allows a reduction of a large Markov chain model of interacting objects to a dynamical system whose state is the occupancy measure (i.e. the distribution of states of all objects), or, more generally, a function of the history of the occupancy measure. The resulting dynamical system is deterministic, but it can be used to study a stochastic system (with considerably smaller dimension) that reflects the state of one or several tagged objects.

\section{References}

[1] L. Afanassieva, S. Popov, and G. Fayolle. Models for transporation networks. Journal of Mathematical Sciences, 1997 - Springer.

[2] F. Baccelli, A. Chaintreau, D. De Vleeschauwer, and D. R. McDonald. Http turbulence, May 2004.

[3] F. Baccelli, M. Lelarge, and D. McDonald. Metastable regimes for multiplexed tcp flows. In Proceedings of the
Forty-Second Annual Allerton Conference on Communication, Control, and Computing, Allerton House, Monticello, Illinois, USA, University of Illinois at Urbana-Champaign, October 2004.

[4] M. Benaim and J. Weibull. Deterministic approximation of stochastic evolution in games. Econometrica, 71(3):873903, 2003.

[5] M.-D. Bordenave, Charles and A. Proutiere. A particle system in interaction with a rapidly varying environment: Mean field limits and applications. arXiv:math/0701363v2.

[6] S. Buchegger and J.-Y. Le Boudec. Performance analysis of the confidant protocol (cooperation of nodes - fairness in dynamic ad-hoc networks). In Proceedings of MobiHoc'02, June 2002.

[7] D. A. Dawson, J. Tang, and Y. Q. Zhao. Balancing queues by mean field interaction. Queueing Systems, 2005.

[8] W. Kang, F. P. Kelly, N. H. Lee, and R. J. Williams. Fluid and Brownian approximations for an Internet congestion control model. In Proceedings of the 43rd IEEE Conference on Decision and Control, 2004.

[9] F. I. Karpelevich, E. A. Pechersky, and Y. M. Suhov. Dobrushin's approach to queueing network theory. Journ. Appl. Math. Stoch. Anal., 9(4):373-398, 1996.

[10] S. Kumar and L. Massoulié. Integrating streaming and filetransfer internet traffic: Fluid and diffusion approximations. MSR-TR-2005-160.

[11] A. Martinoli, K. Easton, and W. Agassounon. Modeling Swarm Robotic Systems: A Case Study in Collaborative Distributed Manipulation. Int. Journal of Robotics Research, 23(4):415-436, 2004. Special Issue on Experimental Robotics, B. Siciliano, editor.

[12] J. Mundinger. Analysis of peer-to-peer systems in communication networks. Ph.D. Thesis, Cambridge University, August 2005.

[13] J. Mundinger and J.-Y. Le Boudec. Analysis of a reputation system for mobile ad-hoc networks with liars. In Proceedings of WiOpt 2005: Modeling and Optimization in Mobile, Ad Hoc and Wireless Networks, pages 41-46, 2005.

[14] J. Mundinger and J.-Y. Le Boudec. Analysis of a robust reputation system for self-organised networks. To appear in European Transactions on Telecommunications, Special Issue on Self-Organisation in Mobile Networking, 16(5), October 2005.

[15] J. Mundinger and J.-Y. Le Boudec. The impact of liars on reputation in social networks. In Proceedings of Social Network Analysis: Advances and Empirical Applications Forum, Oxford, UK, July 2005.

[16] Y. M. Suhov and N. D. Vvedenskaya. Dobrushin's meanfield approximation for a queue with dynamic routing. Markov Processes and Related Fields, 3(4):493-526, 1997.

[17] P. Tinnakornsrisuphap and A. M. Makowski. Limit behavior of ecn/red gateways under a large number of tcp flows. In Proceedings IEEE INFOCOM 2003, The 22nd Annual Joint Conference of the IEEE Computer and Communications Societies, San Franciso, CA, USA, March 30 - April 32003.

[18] B. Ycart. Modèles et algorithmes markoviens, volume 39. Springer Verlag, 2002. 


\section{Appendix}

\subsection{Proof of Theorems 4.1 and 5.1}

We give a combined proof for both theorems. First, we construct a generalization of the fast simulation; we introduce a collection $\tilde{X}_{1}^{N}(t)$ of $N$ interacting objects, obtained by replacing the occupancy measure by its deterministic approximation. Specifically, assume we are given some deterministic occupancy measure $\vec{\mu}(0)$. Define $\tilde{X}_{n}^{N}(t)$ by:

- $\vec{\rho}(t+1)=g(\vec{\rho}(t), \vec{\mu}(t))$ and $\vec{\mu}(t+1)=\vec{\mu}(t) \cdot K(\vec{\rho}(t))$, for $t \geq 0$. In other words, we compute some deterministic $\vec{\rho}(t)$ and $\vec{\mu}(t)$ as in Theorem 5.1.

- At $t=0, \tilde{X}_{n}^{N}(0)=X_{n}^{N}(0)$.

- Call $U_{n}(t)$ the random numbers drawn to simulate $X_{n}^{N}(t)$, i.e., assume that $X_{n}^{N}(t)=$ $\mathcal{P}^{N}\left(X_{n}^{N}(t), \vec{R}^{N}(t), U_{n}\right)\left(U_{n}(s), \quad s=1 \ldots t\right.$, are iid uniform in $(0,1)$ ) and let, for $t \geq 0$ :

$$
\tilde{X}_{n}^{N}(t+1)=\mathcal{P}\left(\tilde{X}_{n}^{N}(t), \vec{\rho}(t), U_{n}(t)\right)
$$

Note that if we would impose $\tilde{X}_{1}^{N}(0)=y(0)$ then we would have $\tilde{X}_{1}^{N}(t)=Y_{1}(t)$. The collection $\tilde{X}_{n}^{N}(t)$ depends on $N$ only because its initial distribution (i.e. at time $t=0)$ does.

Theorem 4.1 follows directly from Lemma 8.3 and Theorem 5.1 from Lemma 8.4.

\subsection{Lemmas}

Lemma 8.1 Let $\tilde{M}^{N}(t)$ be the occupancy measure for $\tilde{X}_{n}^{N}(t)$. Assume $\lim _{N \rightarrow \infty} \vec{M}^{N}(0)=\vec{\mu}(0)$ almost surely. Then for any $t \geq 0, \lim _{N \rightarrow \infty} \tilde{M}^{N}(t)=\vec{\mu}(t)$ a.s.

Proof. By induction on $t$. It is true for $t=0$ by hypothesis and by the fact that $\vec{M}^{N}(0)=\tilde{M}^{N}(0)$. Assume it is true at $t$. Then for any fixed state $j \in \mathcal{E}$ :

$$
\tilde{M}_{j}^{N}(t+1)=\frac{1}{N} \sum_{n=1}^{N} 1_{\left\{\tilde{X}_{n}^{N}(t+1)=j\right\}}=\sum_{i \in \mathcal{E}} V_{i, j}^{N}
$$

with

$$
V_{i, j}^{N}=\frac{1}{N} \sum_{n=1}^{N} 1_{\left\{\tilde{X}_{n}^{N}(t)=i\right\}} 1_{\left\{\mathcal{P}\left(i, \vec{\rho}(t), U_{n}(t+1)\right)=j\right\}}
$$

Consider now a fixed $i$ and let $\theta=K_{i, j}(\vec{\rho}(t)), m=\mu_{i}(t)$. Consider first the case $m=0$. We have then

$$
0 \leq V_{i, j}^{N} \leq \frac{1}{N} \sum_{n=1}^{N} 1_{\left\{\tilde{X}_{n}^{N}(t)=i\right\}}=\tilde{M}_{i}^{N}(t)
$$

and by induction hypothesis $\tilde{M}_{i}^{N}(t) \rightarrow 0$ a.s. Thus, in this case, $\lim _{N \rightarrow \infty} V_{i, j}^{N}=m \theta$ a.s.

We can now assume $m>0$. We apply Lemma 8.2 with $B^{N}=N \tilde{M}_{i}^{N}(t)$ and $\left(Y_{b}^{N}\right)_{b=1 \ldots B^{N}}$ the list of all $1_{\left\{\mathcal{P}\left(i, \vec{\rho}(t), U_{n}(t+1)\right)=j\right\}}$ for $n=1$ to $N$ such that $\tilde{X}_{n}^{N}(t)=i$. With the notation of Lemma 8.2 we have

$$
V_{i, j}^{N}=\frac{B^{N}}{N} Z^{N}
$$

and thus $\lim _{N \rightarrow \infty} V_{i, j}^{N}=m \theta$ a.s. in this case as well.

Using Equation (25), it follows that

$$
\lim _{N \rightarrow \infty} \tilde{M}_{j}^{N}(t+1)=\sum_{i} \mu_{i}(t) K_{i, j}(\vec{\rho}(t))=\mu_{j}(t+1)
$$

Lemma 8.2 For $N \in \mathbb{N}$ let $B^{N}$ be a random integer in the set $\{0,1, \ldots, N\}$, such that almost surely $\lim _{N \rightarrow \infty} \frac{B^{N}}{N}=$ $m>0$. Let $Y_{b}^{N}, b=1 \ldots B^{N}$ be iid Bernoulli random variables, independent of the sequence $B^{N}$, such that $\mathbb{E}\left(Y_{b}^{N}\right)=\theta$. Let $Z^{N}=\frac{1}{B^{N}} \sum_{b=1}^{B^{N}} Y_{b}^{N}$ if $B^{N}>0$ and $Z^{N}=0$ otherwise. Almost surely: $\lim _{N \rightarrow \infty} Z^{N}=\theta$

Proof. Fix some arbitrary $\epsilon>0$. By Cramer's inequality, there exists some fixed $\alpha>0$ such that for any $b \in \mathbb{Z}$ :

$$
\mathbb{P}\left(\left|Z^{N}-\theta\right|>\epsilon \mid B^{N}=b\right) \leq e^{-\alpha b}
$$

Now let $(\Omega, \mathcal{F}, \mathbb{P})$ be the underlying probability space. Let

$\Omega_{n}=\left\{\omega \in \Omega\right.$ such that for all $\left.N \geq n, \frac{B^{N}(\omega)}{N}>\frac{m}{2}\right\}$

Since $\lim _{N \rightarrow \infty} \frac{B^{N}}{N}=m>0$

$$
\mathbb{P}\left(\bigcup_{n \in \mathbb{N}} \Omega_{n}\right)=\lim _{n \rightarrow \infty} \uparrow \mathbb{P}\left(\Omega_{n}\right)=1
$$

Now, by Equation (26),

$$
\begin{aligned}
\mathbb{P}\left(\left|Z^{N}-\theta\right|>\epsilon, \omega \in \Omega_{n}\right) & \leq \mathbb{E}\left(e^{-\alpha B^{N}} 1_{\left\{\omega \in \Omega_{n}\right\}}\right) \\
& \leq e^{-\frac{\alpha m N}{2}} P\left(\Omega_{n}\right)
\end{aligned}
$$

By the Borel-Cantelli lemma, it follows that

$$
P\left(\left|Z^{N}-\theta\right|>\epsilon i . o, \omega \in \Omega_{n}\right)=0
$$

(where i.o denotes infinitely often). By monotone convergence then, $P\left(\left|Z^{N}-\theta\right|>\epsilon i . o\right)=0$ for all $\epsilon$ so $Z^{N} \rightarrow \theta$ a.s. 
Lemma 8.3 Assume $\quad \lim _{N \rightarrow \infty}\left(\vec{M}^{N}(0), \vec{R}^{N}(0)\right)=$ $(\vec{\mu}(0), \vec{\rho}(0))$ almost surely. For any $t \geq 0$, a.s.: $\quad \lim _{N \rightarrow \infty} \frac{1}{N} \sum_{n=1}^{N} 1_{\left\{X_{n}^{N}(t) \neq \tilde{X}_{n}^{N}(t)\right\}}=0$ and $\lim _{N \rightarrow \infty}\left(\vec{M}^{N}(t), \vec{R}^{N}(t)\right)=(\vec{\mu}(t), \vec{\rho}(t))$.

Proof. By induction on $t$. The conclusion is true at $t=0$ by hypothesis and construction of $\tilde{X}_{n}^{N}$. Assume the conclusion holds up to time $t$. We now prove that

$$
\lim _{N \rightarrow \infty} \frac{1}{N} \sum_{n=1}^{N} 1_{\left\{X_{n}^{N}(t+1) \neq \tilde{X}_{n}^{N}(t+1)\right\}}=0 \text { a.s }
$$

We have

$$
\begin{aligned}
& \frac{1}{N} \sum_{n=1}^{N} 1_{\left\{X_{n}^{N}(t+1) \neq \tilde{X}_{n}^{N}(t+1)\right\}}= \\
& \frac{1}{N} \sum_{n=1}^{N}\left(1_{\left\{X_{n}^{N}(t+1) \neq \tilde{X}_{n}^{N}(t+1), X_{n}^{N}(t) \neq \tilde{X}_{n}^{N}(t)\right\}}\right. \\
& \left.\quad+1_{\left\{X_{n}^{N}(t+1) \neq \tilde{X}_{n}^{N}(t+1), X_{n}^{N}(t)=\tilde{X}_{n}^{N}(t)\right\}}\right) \\
& \leq \frac{1}{N} \sum_{n=1}^{N} 1_{\left\{X_{n}^{N}(t) \neq \tilde{X}_{n}^{N}(t)\right\}}+\sum_{i=1}^{S} A_{i}^{N}
\end{aligned}
$$

with

$$
A_{i}^{N}=\frac{1}{N} \sum_{n=1}^{N} 1_{\left\{X_{n}^{N}(t+1) \neq \tilde{X}_{n}^{N}(t+1), X_{n}^{N}(t)=\tilde{X}_{n}^{N}(t)=i\right\}}
$$

The first term in the last inequality converges to 0 almost surely by induction hypothesis; thus, in order to prove Equation (27), all we need is to show that $A_{i}^{N} \rightarrow 0$ a.s.

It follows from Equation (10) that

$$
A_{i}^{N}=\frac{1}{N} \sum_{n=1}^{N} 1_{\left\{U_{n}(t+1) \in I_{i}^{N}\right\}}
$$

where $I_{i}^{N}$ is the union of intervals defined by

$$
\begin{aligned}
& I_{i}^{N}=\bigcup_{j=1}^{S}\left(L_{i, j}^{N}, V_{i, j}^{N}\right] \\
& L_{i, j}^{N}=\min \left(\sum_{j^{\prime}=1}^{j} K_{i, j^{\prime}}^{N}\left(\vec{R}^{N}(t)\right), \sum_{j^{\prime}=1}^{j} K_{i, j^{\prime}}(\vec{\rho}(t))\right) \\
& V_{i, j}^{N}=\max \left(\sum_{j^{\prime}=1}^{j} K_{i, j^{\prime}}^{N}\left(\vec{R}^{N}(t)\right), \sum_{j^{\prime}=1}^{j} K_{i, j^{\prime}}(\vec{\rho}(t))\right)
\end{aligned}
$$

Let $G^{N}()$ be the empirical distribution function of the $N$ uniforms $U_{n}(t+1), n=1, \ldots, N$, i.e. $G^{N}(x)=$ $\frac{1}{N} \sum_{n=1}^{N} 1_{\left\{U_{n}(t+1) \leq x\right\}}$. Then

$$
A_{i}^{N} \leq \sum_{j}\left(G^{N}\left(V_{i, j}^{N}\right)-G^{N}\left(L_{i, j}^{N}\right)\right)
$$

Hence

$$
\begin{gathered}
A_{i}^{N} \leq \sum_{j}\left(\left|G^{N}\left(V_{i, j}^{N}\right)-V_{i, j}^{N}\right|+\left|G^{N}\left(L_{i, j}^{N}\right)-L_{i, j}^{N}\right|\right. \\
\left.\quad+\left|V_{i, j}^{N}-L_{i, j}^{N}\right|\right) \\
\leq 2 S \sup _{x \in[0,1]}\left|G^{N}(x)-x\right|+\sum_{j}\left|V_{i, j}^{N}-L_{i, j}^{N}\right|
\end{gathered}
$$

By the Glivenko-Cantelli lemma

$$
\lim _{N \rightarrow \infty} \sup _{x \in[0,1]}\left|G^{N}(x)-x\right|=0 \text { a.s. }
$$

thus the first term in Equation (29) converges to 0 a.s. Now by induction hypothesis, $\vec{R}^{N}(t) \rightarrow \vec{\rho}(t)$, and thus, by hypothesis $\mathbf{H},\left|V_{i, j}^{N}-L_{i, j}^{N}\right| \rightarrow 0$ a.s. This shows Equation (27).

Next:

$$
\begin{aligned}
& \left\|\vec{M}^{N}(t+1)-\vec{\mu}(t+1)\right\| \leq\left\|\vec{M}^{N}(t+1)-\tilde{M}^{N}(t+1)\right\| \\
& +\left\|\tilde{M}^{N}(t+1)-\vec{\mu}(t+1)\right\|
\end{aligned}
$$

where we can take any norm since $\vec{M}^{N}(t)$ is in a space of finite dimension. We take the $L^{1}$ norm (which is, up to a constant, the same as the norm of total variation): $\|\vec{m}\|=\sum_{i}\left|m_{i}\right|$. The latter term in the right hand-side of Equation (30) converges to 0 a.s. by Lemma 8.1. As to the former:

$$
\begin{aligned}
& \left\|\vec{M}^{N}(t+1)-\tilde{M}^{N}(t+1)\right\| \\
& =\sum_{i}\left|M_{i}^{N}(t+1)-\tilde{M}_{i}^{N}(t+1)\right| \\
& =\frac{1}{N} \sum_{i}\left|\sum_{n=1}^{N} 1_{\left\{X_{i}^{N}(t+1)=i\right\}}-1_{\left\{\tilde{X}_{i}^{N}(t+1)=i\right\}}\right| \\
& \leq \frac{1}{N} \sum_{n=1}^{N} \sum_{i}\left|1_{\left\{X_{i}^{N}(t+1)=i\right\}}-1_{\left\{\tilde{X}_{i}^{N}(t+1)=i\right\}}\right| \\
& \leq \frac{2}{N} \sum_{n=1}^{N}\left|1_{\left\{X_{i}^{N}(t+1) \neq \tilde{X}_{i}^{N}(t+1)\right\}}\right|
\end{aligned}
$$

thus it also converges to 0 a.s. This shows that $\lim _{N \rightarrow \infty} \vec{M}^{N}(t+1)=\vec{\mu}(t+1)$ a.s.; by continuity of $g()$, it follows that $\lim _{N \rightarrow \infty} \vec{R}^{N}(t+1)=\vec{\rho}(t+1)$ a.s. as well.

Lemma 8.4 Assume $\quad \lim _{N \rightarrow \infty}\left(\vec{M}^{N}(0), \vec{R}^{N}(0)\right)=$ $(\vec{\mu}(0), \vec{\rho}(0))$ a.s. For any fixed $t$, almost surely there exists some random number $N_{0}$ such that, for $N \geq N_{0}$ $\tilde{X}_{1}^{N}(s)=X_{1}^{N}(s)$ for all $s=0, \ldots, t$.

Proof. Let $\mathcal{X}$ be the set of numbers in $x \in[0,1]$ that can be written $x=\sum_{j^{\prime}=1}^{j} K_{i, j^{\prime}}(\rho(s))$ for some $i, j$ and $s$. Since $\mathcal{X}$ is enumerable, its Lebesgue measure is 0 and thus, almost surely, $U_{1}(s) \notin \mathcal{X}$ for all $s$.

The proof now proceeds by induction on $t$. The conclusion is true at $t=0$; assume now it is true up to $t$. Then for $N \geq N_{0}, \tilde{X}^{N}(t)=X^{N}(t)=i$. Let $j=\tilde{X}^{N}(t+1)$. We can assume $U_{1}(t+1) \notin \mathcal{X}$ thus

$$
\sum_{j^{\prime}=1}^{j-1} K_{i, j^{\prime}}(\vec{\rho}(t))<U_{1}(t+1)<\sum_{j^{\prime}=1}^{j} K_{i, j^{\prime}}(\vec{\rho}(t))
$$

By Lemma $8.3, \lim _{N \rightarrow \infty} \vec{R}^{N}(t)=\vec{\rho}(t)$ a.s. and by hypothesis $\mathbf{H}$ there exists $N_{1} \geq N_{0}$ such that

$$
\sum_{j^{\prime}=1}^{j-1} K_{i, j^{\prime}}^{N}\left(\vec{R}^{N}(t)\right)<U_{1}(t+1)<\sum_{j^{\prime}=1}^{j} K_{i, j^{\prime}}^{N}\left(\vec{R}^{N}(t)\right)
$$

and thus $X_{1}^{N}(t+1)=j=\tilde{X}_{n}^{N}(t+1)$ for $N_{1} \geq N_{0}$. 